\title{
FRF-based Structural Damage Detection Using Kohonen Self-organising Maps
}

\author{
C. Zang and M. Imregun \\ Imperial College of Science, Technology \& Medicine, Mechanical Engineering Department, London SW7 2BX, UK
}

(Received 16 May 2000; accepted 18 September 2000)

\begin{abstract}
This paper presents a structural damage detection method using measured frequency response functions (FRFs). The size of the raw vibration data was reduced via a principal component analysis. Kohonen neural networks, the so-called self-organising maps because of their ability to function without supervised training, were used for the actual damage detection. The procedure has two distinct steps. The first step consists of determining the principal components of a response variation matrix formed by including all available FRF data. Substantial data compression is then achieved by projecting the FRFs onto their most significant principal components. During the second step, the compressed data are fed into a Kohonen artificial neural network where the input layer is directly connected to the output layer. Using a winning neuron algorithm for each available measurement, the FRFs are automatically grouped into distinct clusters according to the dynamic properties of the structure they represent, a feature that provides a straightforward detection method. The methodology was applied to 100 measured FRFs obtained from healthy and damaged railway wheels, each response function having 4,096 spectral lines. The visualisation of a $10 \times 10$ Kohonen output layer showed that the FRFs from the damaged and healthy specimens were grouped in distinct clusters with no overlaps. It was concluded that the approach was well suited to on-line industrial applications, not only because of its ability to work with limited measurement data, but also because of the automatic damage detection capability of Kohonen networks, without requiring any supervised training.
\end{abstract}

\section{INTRODUCTION}

Damage detection and structural health monitoring are becoming increasingly important for uninterrupted operation of industrial processes and for ensuring safe operating environments. More critically, high-cost structures, built for civil engineering and aerospace applications, have stringent inspection requirements in order to prevent catastrophic failures and to prolong their in-service lifetime. Recent advances in data acquisition and numerical modelling techniques have resulted in a variety of new techniques and detailed surveys are given by Doebling et al. ${ }^{1,2}$ and Friswell \& Penny ${ }^{3}$. Here we are primarily concerned with damage identification techniques using low-frequency vibration tests. Non-destructive testing techniques, such as ultrasonic guided waves (Alleyne et $a .^{4}$ ), are well outside the scope of this paper. Generally speaking, the damage detection techniques can be classified according to the type of measured data on which they are based. Lew and Sathananthan ${ }^{5}$ used time domain data to compare three different damage detection methods. Cattarius and Inman ${ }^{6}$ also used a time-domain analysis for damage detection in smart structures. Measured frequency response functions (FRFs) were used by Maia et al. ${ }^{7}$ and Sampaio et al. ${ }^{8}$ Most damage identification algorithms dealing with FRFs use a validated base model, usually developed from a finite element formulation, and attempt to find discrepancies between this reference model and the FRFs of some damaged specimen (Fritzen and Bohle'; Wang, Lin and Lim ${ }^{\mathbf{1 0}}$; Thyagarajan et al. $\left.{ }^{11}\right)$. Such an approach may be considered to be an extension of the finite element model updating methodology to damage detection (Friswell \& Mottershead ${ }^{12}$ ). As an alternative approach, modal parameter based methods were used by Morassi and Rovere ${ }^{13}$ who located a notch in a steel frame by considering natural frequency changes between the healthy and damaged states of the structure. Contursi et al. ${ }^{14}$ put forward a multiple damage location assurance criterion, also based on natural frequency changes. Rizos et al. ${ }^{15}$ used the vibration modes to identify the crack location and size in a cantilever beam. Topole and Stubbs ${ }^{16}$ used the mode shapes to detect damage in complex structures. Lam et al. ${ }^{17}$ investigated the identification of damaged structural connections using experimentally-derived modal parameters and sensitivity analysis. Broadly speaking, damage detection methods based on vibration data suffer from a number of drawbacks such as measurement noise, practical difficulties of obtaining a reference model for comparison purposes, inaccuracies in modal analysis and the insensitivity of the modal parameters to local mass and stiffness changes.

More recently, techniques based on multivariate statistics and neural networks have been applied to structural healthy monitoring and damage detection (Doebling et al. ${ }^{1}$, Zeng ${ }^{18}$ ). However, there are two main problems associated with such a route. The first one is the difficulty of handling the size of the measured data in a neural network context, referred to as 'the curse of dimensionality' (Bishop ${ }^{19}$ ). The measured FRFs may contain thousands of spectral lines and the direct use of such data will require as many neural network input nodes, a constraint which makes the number of training samples impractically large. To circumvent the problem, Atalla and Inman $^{20}$, and Levin and Lieven ${ }^{21}$ used preselected data points and modal analysis respectively, though some loss of useful data was inevitable. The second difficulty is the requirement to train the networks using a sufficient number of training samples. Indeed, back-propagation and radial basis-function networks have been proven to be effective for damage detec- 